\title{
Two-sided variable inspection plans for arbitrary continuous populations with unknown distribution
}

\author{
Wolfgang Kössler • Janine Ott
}

Received: date / Accepted: date

\begin{abstract}
The ordinary variable inspection plans rely on the normality of the underlying populations. However, this assumption is vague or even not satisfied. Moreover, ordinary variable sampling plans are sensitive against deviations from the distribution assumption.

Nonconforming items occur in the tails of the distribution. They can be approximated by a Generalized Pareto distribution (GPD). We investigate several estimates of their parameters according to their usefulness not only for the GPD, but also for arbitrary continuous distributions. The Likelihood Moment estimates (LME) of Zhang (2007) and the Bayesian estimate (ZSE) of Zhang and Stephens (2009) turn out to be the best for our purpose. Then we use these parameter estimates to estimate the fraction defective.

The asymptotic normality of the LME (cf. Zhang, 2007) and of the fraction defective are used to construct the sampling plan. The difference to the sampling plans constructed in Kössler $(1999,2015)$ is that we now use the new parameter estimates. Moreover, in contrast to the aforementioned papers, we now also consider two-sided specification limits.

An industrial example illustrates the method.

Keywords Extreme value index · Fraction defective $\cdot$ Generalized Pareto distribution $\cdot$ Peak over Threshold method $\cdot$ Likelihood Moment estimate $\cdot$ Zhang-Stephens estimate

Mathematics Subject Classification (2000) MSC 62F10 - 62F12 - 62G30 - 62G32 . 62P30

W. Kössler

Humboldt-Universität zu Berlin, Department of Computer Science, Rudower Chaussee 25, D-12489 Berlin Tel.: +49-30-20933087

Fax: +49-30-20933932

E-mail: koessler@informatik.hu-berlin.de

J. Ott

Humboldt-Universität zu Berlin, Department of Computer Science, Rudower Chaussee 25, D-12489 Berlin
\end{abstract}

This is a post-peer-review, pre-copyedit version of an article published in AStA Advances in Statistical Analysis.

The final authenticated version is available online at:

https://doi.org/10.1007/s10182-018-00338-w 


\section{Introduction}

We consider a lot of units having a quality characteristic $X$ with a (unknown) continuous cumulative distribution function (cdf) $F$. Given there are lower and upper specification limits $L$ and $U$ the fraction defective $p$ of the lot is defined by

$$
p=P(X<L)+P(X>U)=F(L)+1-F(U) .
$$

A decision is to be made whether the lot is to be accepted or not. This decision is made on hand of a sample. The sample size $n$ is to be computed in advance. Then a suitable statistic $S$ based on the sample $X_{1}, \ldots, X_{n}$ of size $n$ and an acceptance number $c$ are chosen such that predetermined conditions are satisfied. It makes sense to use the statistic $S=\hat{p}$, i.e. an reasonable estimate of the fraction defective $p$. Our estimate will be based on the $m(2 m<n)$ smallest and largest observations, respectively. Our variable inspection plan $(n, m, c)$ is then defined by: if $\hat{p} \leq c$ the lot will be accepted else it will be rejected. The sample size $n$, the subsample size $m$ and the acceptance number $c$ are to be determined. The operating characteristic (OC) denoted by $L^{n, m, c}(p)$ is the probability of accepting the lot, i.e.

$$
L^{n, m, c}(p):=P_{p}(\hat{p} \leq c), \quad 0<p<0.5,
$$

and it depends, of course, on the sampling plan $(n, m, c)$ and it is a function of the fraction defective.

Variable inspection plans are computed by minimizing the sample size $n$ while meeting the "2-point conditions"

$$
\begin{aligned}
& L^{n, m, c}\left(p_{1}\right) \geq 1-\alpha \\
& L^{n, m, c}\left(p_{2}\right) \leq \beta
\end{aligned}
$$

$\left(0<p_{1}<p_{2}<1,0<\beta<1-\alpha\right)$, where $p_{1}$ and $p_{2}$ are the accepted or rejected quality limits and stay for good or bad quality, respectively.

The conditions (1) and (2) reflect the desires of the producer (who delivers the lot) and the consumer (who gets the lot). Good quality should be accepted with high probability, at least $1-\alpha$, and bad quality should be accepted with low probability, at most $\beta$. The four values $p_{1}, p_{2}, \alpha, \beta$ are agreed by producer and consumer in advance.

In the case of a normally distributed quality characteristic $X$ the LiebermanResnikoff plan (LR-plan, cf. Resnikoff, 1952, Lieberman and Resnikoff, 1955, BruhnSuhr and Krumholz, 1991) or the Maximum-Likelihood plan (ML-plan, cf. BruhnSuhr and Krumbholz, 1990), respectively, can be applied.

Algorithms to determine exact ML- or LR-plans are implemented in the programs ExVar, cf. Kössler et al (1994) and ExLiebRes, cf. Krumbholz and Steuer (2014). However, these inspection plans are very sensitive with respect to deviations from the normal distribution assumption (cf. Kössler and Lenz, 1995,1997) .

This problem gives rise to the question what is to do in the case of not normal or unknown cdfs? One way is to perform sampling inspection by attributes where an item $X_{i}$ is considered to be nonconforming if and only if $X_{i}<L$ or $X_{i}>U$. The OC is easy to compute (cf. Uhlmann, 1982). However, this method requires relatively large sample sizes. 
In two articles (Kössler, 1999, 2015) we introduced one-sided variable inspection plans which are based on approximating the tails of the underlying density by a Generalized Pareto distribution (GPD), cf. e.g. Pickands (1975) and Smith (1987).

In Kössler (1999) we assumed that the population has long to medium tails and used the Maximum Likelihood estimators (MLE) for the parameters of the GPD. In the other article (Kössler, 2015) we assumed short or medium tails and used modified Smith-Weissman (1985) estimates (SWM). Both estimates are biased, therefore we need a correction of the acceptance number $c$.

In the current paper, we use the parameter estimates of Zhang (2007) and Zhang and Stephens (2009) that turn out to be better in practice than the MLE and SWM. In contrast to the MLE and SWM the new parameter estimates can be applied to arbitrary continuous distributions. They are now used to estimate the fraction defective.

Moreover, we generalize the one-sided variable inspection plans to the case of both, lower and upper specification limits.

In section 2 we recall the variable inspection plans proposed in Kössler (1999, 2015) which are based on the asymptotic normality of the estimates of the fraction defective. Various methods of parameter estimates are investigated in section 3. It turns out that the Likelihood moment estimate (LME) of Zhang (2007) and the Zhang-Stephens estimate (ZSE) of Zhang and Stephens (2009) using a Bayesian approach behave best. The LME is asymptotically normal (Zhang, 2007) but as we will see it is biased (cf. also Mackay et al. (2011)). For the ZSE the proof of consistency and asymptotic normality seems to be very complicated. Both estimators, LME and ZSE, have the advantage that there are, except continuity and not very short tails, no restrictions on the underlying distribution. In section 4 we examine whether the inspection plans constructed in Kössler $(1999,2015)$ can be used, but now in the case of double specification limits.

In simulation studies performed in section 5 we first check the finite sample behaviour of the most promising parameter estimates LME and ZSE. Then we compute the OC of our sampling plan using these estimates and investigate how good the asymptotic theory works. However, we will need a relatively large sample size. The sample sizes needed are still less than that for attribute plans.

The results are in accordance with those of the corresponding one-sided sampling plans for medium to long tails, cf. Kössler (1999) and with those for short tails cf. Kössler (2015) but with the advantages that we need only one estimate in the whole parameter space. If the Zhang and Stephens estimator (ZSE) is used we also do not need any correction of the acceptance number.

Our method is illustrated and discussed in section 6. We conclude with a short summary.

\section{Extension of the variable sampling plans proposed in Kössler $(1999,2015)$}

In this section we briefly summarize the construction of our variable sampling plans. Then we extend them to the case of double specification limits.

Recall, the main idea is that nonconforming items $X_{i}$ occur in the tails of the underlying cdf., namely $X_{i}<L=F^{-1}\left(p_{L}\right)$ or $X_{i}>U=F^{-1}\left(1-p_{U}\right)$ with 
the (unknown) fraction defective $p=p_{L}+p_{U}$. Additionally, items $X_{i}$ with $X_{i} \approx$ $L, X_{i}>L$ or $X_{i} \approx U, X_{i}<U$ can be considered suspicious. They also should be taken into account in inspection plans. These thoughts give rise to the idea that we use two thresholds $t_{U}$ and $t_{L}, L<t_{L}<t_{U}<U$. All observations $X_{i}$ with $X_{i}<t_{L}$ or $X_{i}>t_{U}$ are used. It is convenient to determine these thresholds by some $q$-quantile and $(1-q)$-quantile, respectively, where $q$ is to be fixed in advance, see below.

The tails of a continuous distribution may be approximated by a Generalized Pareto distribution (GPD) function which is defined by

$$
G P D(y ; \sigma, k):= \begin{cases}1-\left(1-\frac{k y}{\sigma}\right)^{\frac{1}{k}} & \text { if } k \neq 0 \\ 1-e^{-\frac{y}{\sigma}} & \text { if } k=0\end{cases}
$$

where $k$ is a shape parameter and $\sigma>0$ is a scale parameter. The range of the GPD is given by $0<y<\infty$ if $k \leq 0$ and $0<y<\frac{\sigma}{k}$ if $k>0$ (cf. e.g. Smith, 1987, p.1175). The parameters $k$ and $\sigma(t)$ are given by the extreme value distribution theory, cf. e.g. Falk (1987) or Kössler (1999). Note that the parameter $-k$ is sometimes called the extreme value index of the underlying distribution, cf. de Haan and Fereira (2006).

Consider first the upper tail. Let $t=t_{U}$ be an arbitrary real value of the support of the cdf $F$ and $x_{U}:=\sup _{x \in \text { suppF }} x$ where $x_{U}=\infty$ is admissible.

The conditional distribution $F_{t}^{U}(y)$ of $X-t$ conditioned under $X>t$ is given by

$$
F_{t}^{U}(y)=\frac{F(t+y)-F(t)}{1-F(t)},
$$

where $0<y<x_{U}-t$. If $t \rightarrow x_{U}$ this distribution converges uniformly to a GPD with certain parameters $\sigma_{U}$ and $k_{U}$ as was shown by Balkema and de Haan (1974) and Pickands (1975, Theorem 7), i.e.

$$
\lim _{t \rightarrow x_{U}}\left\|F_{t}^{U}(y)-G P D\left(Y ; \sigma_{U}, k_{U}\right)\right\|_{\infty}=0 .
$$

Similarly we consider the lower tail. Let $t=t_{L}<t_{U}$ be an arbitrary real value of the support of the cdf $F$, and $x_{L}:=\inf _{x \in \operatorname{supp} F} x$ where $x_{L}=-\infty$ is admissible.

The conditional distribution $F_{t}^{L}(y)$ of $t-X$ conditioned under $X<t$ is given by

$$
F_{t}^{L}(y)=\frac{F(t)-F(t-y)}{F(t)},
$$

where $0<y<t-x_{L}$. If $t \rightarrow x_{L}$ this distribution again converges uniformly to a GPD with certain parameters $\sigma_{L}$ and $k_{L}$.

Of course, by using the GPD, we use approximations of the true (upper and lower) fraction defective only. However, simulations performed in Kössler (1999) and in Ott (2016) show that the approximation error is rather small.

Let $q$ be given, $0<q<0.5$, for our choice of $q$ see the next two paragraphs. We define two thresholds, $t_{L}$ and $t_{U}$ by $t_{L}=F^{-1}(q)$ and $t_{U}=F^{-1}(1-q)$, respectively. Then we estimate them by $\hat{t}_{L}=X_{(m+1)}$ and $\hat{t}_{U}=X_{(n-m)}$, where $m:=\lfloor n q\rfloor$ and $X_{(i)}$ denotes the $i$ th order statistics of the sample. 
There are some papers that discuss the choice of thresholds, cf. e.g. Caeiro and Gomes (2015) and the references therein. However, they are all data-driven, i.e. the threshold is based on the underlying sample. Such approaches are not possible here since we have to develop a procedure that defines the value of $q$ (which determines the threshold) in advance in order to minimize the sample size later on.

For a discussion of the choice of the fraction $q$ in advance, we refer to Kössler $(1999,2015)$. Our choice is

$$
q=q\left(n_{0}\right)=p_{2}+\frac{1}{\sqrt{n_{0}}},
$$

where $p_{2}$ is the rejected quality limit, and $n_{0}$ is the initial sample size obtained by averaging the sample sizes $n_{V}$ and $n_{A}$ of the ordinary variable sampling plan and the attribute sampling plan, $n_{0}=\frac{n_{V}+n_{A}}{2}$. This definition reflects the conditions $q>p, q \rightarrow 0$ (often we have a condition similar to $q=\mathcal{O}\left(\frac{1}{\sqrt{n}}\right)$ or $q=o\left(\frac{1}{\sqrt{n}}\right)$ ) and also that we expect the resulting sample size to lie between $n_{V}$ and $n_{A}$.

Let $\hat{y}_{L}=\hat{t}_{L}-L, \hat{y}_{U}=U-\hat{t}_{U}$, and $\left(\hat{k}_{L}, \hat{\sigma}_{L}\right)$ and $\left(\hat{k}_{U}, \hat{\sigma}_{U}\right)$ be consistent estimates of $\left(k_{L}, \sigma_{L}\right)$ and $\left(k_{U}, \sigma_{U}\right)$, respectively, in the GPD-model. Then

$$
\begin{aligned}
& \hat{p}_{L}=q \cdot\left\{\begin{array}{lll}
\left(1-\frac{\hat{k}_{L} \hat{y}_{L}}{\hat{\sigma}_{L}}\right)^{\frac{1}{\hat{k}_{L}}} & \text { if } & \hat{k}_{L} \neq 0 \\
e^{-\frac{\hat{y}_{U}}{\hat{\sigma}_{L}}} & \text { if } & \hat{k}_{L}=0
\end{array}\right. \text { and } \\
& \hat{p}_{U}=q \cdot\left\{\begin{array}{lll}
\left(1-\frac{\hat{k}_{U} \hat{y}_{U}}{\hat{\sigma}_{U}}\right)^{\frac{1}{\hat{k}_{U}}} & \text { if } & \hat{k}_{U} \neq 0 \\
e^{-\frac{\hat{y}_{U}}{\hat{\sigma}_{U}}} & \text { if } & \hat{k}_{U}=0
\end{array}\right. \\
& \hat{p}=\hat{p}_{L}+\hat{p}_{U}
\end{aligned}
$$

are consistent estimates of $p_{L}, p_{U}$, and $p$, respectively. The consistency follows from the continuity of the functions $p_{j}=p_{j}\left(k_{j}, \sigma_{j}, t_{j}\right), j=U, L$ and from the Balkemade Haan-Pickands theorem, cf. (4). Recall that for the latter we need $t_{U} \rightarrow x_{U}$ and $t_{L} \rightarrow x_{L}$.

Note that $\hat{y}_{L}$ and $\hat{y}_{U}$ are random, and the estimates (6) and (7) are well defined if $\hat{y}_{L}, \hat{y}_{U} \geq 0$ and if

$$
\hat{k}_{L} \hat{y}_{L}<\hat{\sigma}_{L} \quad \text { and } \quad \hat{k}_{U} \hat{y}_{U}<\hat{\sigma}_{U} .
$$

In the few cases in that $\hat{y}_{L}<0$ or $\hat{y}_{U}<0$ we may reject the lot without further computations because these cases indicate low quality.

All estimates considered in section 3 satisfy the condition (9) if $X_{(1)}<L$ and $X_{(n)}>U$. The cases $X_{(1)} \geq L$ and $X_{(n)} \leq U$ indicate good quality in the lower and upper tail, respectively, and therefore we may set $\hat{p}_{L}:=0$ and $\hat{p}_{U}:=0$, respectively. For example, the probability $P\left(L \leq X_{(1)}<X_{(n)} \leq U\right)=(1-p)^{n}$ is small for a rather large fraction defective $p$ and sample size $n$, and therefore we will have only a small negative bias introduced in the estimate of $p$ by setting $\hat{p}=0$.

Note also that the cases $L=-\infty$ or $U=\infty$ are admissible. Then the general case reduces to the case with one-sided specification limits.

For the construction of the sampling plan, we use the asymptotic normality of the tail estimator $\hat{p}$ given by (8), $\sqrt{m} \frac{\hat{p}-p}{p} \rightarrow \mathcal{N}(0, W)$ with variance $W=W(p)$, which 
depends on the true fraction defective $p$ but also on $q$. For a short discussion of the variance term we refer to section 4 .

Let $\Phi^{-1}($.$) be the quantile function of the standard normal. Our variable inspec-$ tion plan is a solution of the two-point condition inequalities with minimal sample size $n$ (cf. Kössler, 1999, 2015),

$$
\begin{aligned}
m & =\left\lceil\frac{1}{\left(p_{1}-p_{2}\right)^{2}}\left(p_{2} \sqrt{W\left(p_{2}\right)} \Phi^{-1}(\beta)-p_{1} \sqrt{W\left(p_{1}\right)} \Phi^{-1}(1-\alpha)\right)^{2}\right\rceil \\
c & =p_{1}+\Phi^{-1}(1-\alpha) \frac{p_{1} \sqrt{W\left(p_{1}\right)}}{\sqrt{m}} \\
n & =\left\lceil\frac{m}{q}\right\rceil .
\end{aligned}
$$

Recall, $n$ denotes the full sample size, $m$ is the number of the smallest and largest observations used and $c$ is the acceptance number which often is to be modified because of bias.

Since the subsample size $m$ is essential we denote our sampling plan, different to the ordinary sampling plans, by a triple $(n, m, c)$. Recall, the lot is accepted iff $\hat{p} \leq c$ where the estimate $\hat{p}$ is based on the $m$ smallest and largest observations, respectively, of the sample of size $n$.

\section{Comparison of the parameter estimates}

In the literature, there are various proposals for estimates of the parameters of the GPD. We consider the moment estimate (MOM), two variants of the probability weighted moment estimate (PWM), that of Hosking and Wallis (1987) as well as that of Beirlant et al. (2004), the Maximum Likelihood estimate (MLE), with the implementation of Grimshaw (1993), the expectile percentile method (EPM) of Castillo and Hadi (1997), a modified Smith and Weissman (1985) estimate (SWM), as well as the more recent Likelihood moment estimate (LME) and Bayesian estimate (ZSE) of Zhang (2007) and Zhang and Stephens (2009), respectively. For the MOM, PWM, MLE and EPM we refer to Beirlant (2004) and the references therein. The asymptotic variances of the MOM and PWM estimates are much larger than that of the MLE. Moreover, our simulation studies which we performed by SAS programs show that the empirical variances of the resulting estimates of the fraction defective are much larger if the MOM or PWM or the EPM estimates are used. These results are in accordance with that of Beirlant et al. (2004) and Zhang (2007).

In the case of a GPD with parameter values of $k \leq 0.5$ the MLE are optimal and asymptotically normal. Under the extreme value condition, i.e. not necessary exact GPD, they are consistent, under the second order condition they are also asymptotically normal if $k<1$. For $k>1$ the MLE is inconsistent (Zhou, 2009, 2010). In their original version (Smith, 1987, Grimshaw, 1993) the MLE is biased (Kössler, 1999, Beirlant et al., 2004) and sometimes difficult to compute. Some attention was taken to find bias corrected versions of the MLE. In the recent paper of Giles et al. (2016) the authors found a bias correction for the MLE that works good for the parameter 
values of $k<0.3$. Observe, that the bias correction term is of order $\mathcal{O}\left(\frac{1}{n}\right)$, the same that we used in our former sampling plan (see Kössler, 1999). For values of $k>0.3$ Giles et al (2016) propose to use the LME.

Other proposals of bias corrected estimates are summarized in Beirlant et al (2012). They are restricted to some (wide) subclasses of densities, most of them assume heavy tails.

On the other hand, the SWM estimates are suitable if $k \geq 0.5$, i.e. in the case of light tails, cf. Kössler (2015).

The LME is relatively simple, but it is also biased. In contrast to the other estimators the ZSE seems to be unbiased. Therefore, no correction of the acceptance number will be necessary.

In the following two subsections we summarize the most promising estimates LME and ZSE.

We consider the upper tail. For the lower tail the derivation is analogous. Let $y_{i}=X_{(n-m+i)}-X_{(n-m)}, i=1, \ldots, m$ be the shifted observations, shifted by the threshold value, in the upper tail.

3.1 Likelihood moment estimate (LME) of Zhang (2007)

Zhang (2007) combines the Maximum Likelihood method and the moment method and he obtains a relatively simple estimate, which avoids disadvantages of the single methods, as they do not work for short or long tails, respectively.

To obtain the estimate, the reparametrisation from $(\sigma, k)$ to $(\theta, k)$ with $\theta=\frac{k}{\sigma}$ which was proposed by Davison (1984) is used. Let $\mathbf{Y}=\left(y_{1}, \ldots, y_{m}\right)$. From the log-Likelihood function

$$
l_{Z}(\mathbf{Y} ; \theta, k)=m \log \frac{\theta}{k}+\left(\frac{1}{k}-1\right) \sum_{i=1}^{m} \log \left(1-\theta y_{i}\right)
$$

the partial derivatives according $\theta$ and $k$ are taken and set to zero. One of the equations obtained is

$$
k=-\frac{1}{m} \sum_{i=1}^{m} \log \left(1-\theta y_{i}\right), \quad \theta<\frac{1}{y_{m}} .
$$

The other idea is to use the moment equation $(Y \sim G P D(\sigma, k))$

$$
\mathbf{E}(1-\theta Y)^{r}=(1+r k)^{-1}
$$

for arbitrary $r$ with $1+r k>0$.

Replacing $r$ in (14) by $-\frac{r}{k}$, estimating the expectation and putting the explicit expression for $k$ in the resulting equation one obtains the nonlinear equation in $\theta$,

$$
\frac{1}{m} \sum_{i=1}^{m}\left(1-\theta y_{i}\right)^{s}-\frac{1}{1-r}=0
$$


with $s=r m\left(\sum_{i=1}^{m} \log \left(1-\theta y_{i}\right)\right)^{-1}$, which may quite easily be solved by the Newton-Raphson method. Inserting the resulting estimate $\hat{\theta}$ in the equations for $k$ and $\sigma$ yields the LME for $(k, \sigma)$. Note that we have excluded the case $\theta<\frac{1}{y_{m}}$.

Inserting these estimates $(\hat{\sigma}, \hat{k})$ in (6) and (7) we obtain the LME $\hat{p}_{U}$ or $\hat{p}_{L}$ for the upper or lower parts of the fraction defective, respectively.

In the case of $k<\frac{1}{2}$ the asymptotic normality of the estimates is established in Theorem 2.2 of Zhang (2007),

$$
\sqrt{m}\left(\begin{array}{c}
\hat{\sigma} / \sigma-1 \\
\hat{k}-k
\end{array}\right) \rightarrow_{D} \mathcal{N}(\mathbf{0}, \mathbf{\Sigma})
$$

where

$$
\boldsymbol{\Sigma}=\left(\begin{array}{cc}
2+\frac{(r+k)^{2}-2 k}{1-2 r} & 1+\frac{r^{2}+k^{2}-k}{1-2 r} \\
1+\frac{r^{2}+k^{2}-k}{1-2 r} & (1-r)\left(1+\frac{2 k^{2}-2 k+r}{1-2 r}\right)
\end{array}\right), \quad r, k<\frac{1}{2}
$$

Zhang (2007) suggested to choose the free parameter $r=-0.5$, and in our simulation we followed this suggestion. Note that Mackay et al. (2011) investigated several variants of LME but they found no improvements of it. (Following the proof of Theorem 2.2 of Zhang (2007) we see that the case $k=\frac{1}{2}$ is also admissible.)

The proof of the asymptotic normality of $\hat{p}_{U}$ and $\hat{p}_{L}$ follows the same line as in Smith $(1987$, ch.8,9), cf. Kössler $(1999,2015)$, where in the variance term the asymptotic covariance matrix is to be replaced by (15).

We assume $k \leq 0.5,\left(k=k_{L}, k_{U}\right)$, i.e. the cdf. $F$ has not too short (lower respectively upper) tails. For comparison, if $F$ is a triangle cdf. then we have $k_{U}=$ 0.5 .

Under certain conditions on the convergence of $t \rightarrow x_{o}, U \rightarrow x_{o}$ if $n \rightarrow \infty$ the LME $\hat{p}_{U}$ is asymptotically normally distributed with expectation zero and variance $V\left(p_{U}\right)$.

$$
\sqrt{m} \frac{\hat{p}_{U}-p_{U}}{p_{U}} \rightarrow_{D} \mathcal{N}\left(0, V\left(p_{U}\right)\right) .
$$

A computation of the asymptotic variances of the LME shows that they are similar for different distributions. Moreover, they are similar to that obtained if the MLE or SWM is used. A table of asymptotic variances if the MLE are used can be found in Kössler (1999).

\subsection{The method of Zhang and Stephens (2009)}

The method proposed by Zhang and Stephens (2009) is a Bayes estimate, where the parameter $\theta$, cf. the reparametrisation introduced in the previous section, is considered random with a priori distribution GPD, $\theta \sim G P D\left(k^{\prime}, \sigma^{\prime}\right), k^{\prime}=-0.5, \sigma^{\prime}=$ $\left(6 y_{\left(\left\lfloor\frac{m}{4}+0.5\right\rfloor\right)}\right)^{-1}$. The parameters $k^{\prime}$ and $\sigma^{\prime}$ suggested by Zhang and Stephens (2009) are motivated by simulation studies. 


$$
\begin{aligned}
& \text { Let } m^{*}=20+\lfloor\sqrt{m}\rfloor \\
& \qquad \theta_{j}=\frac{1}{y_{(m)}}+\frac{1-\sqrt{\frac{m^{*}}{j-0.5}}}{3 y_{\left(\left\lfloor\frac{m}{4}+\frac{1}{2}\right\rfloor\right)}} \quad \text { and } \quad \omega\left(\theta_{j}\right)=\frac{1}{\sum_{i=1}^{m^{*}} e^{l\left(Y, \theta_{i}\right)-l\left(Y, \theta_{j}\right)}},
\end{aligned}
$$

where $l(Y, \theta)=m \log \frac{\theta}{k}+k-1$ with $k$ given by (13) is the profile log-Likelihood. The resulting estimate is then given by

$$
\hat{\theta}=\sum_{j=1}^{m^{*}} \theta_{j} \omega\left(\theta_{j}\right) .
$$

Inserting the estimate (17) in the equations for $k$ and $\sigma$ yields the Zhang-Stephens estimator ZSE for $(k, \sigma)$.

For shape parameters $-1 \leq k \leq 1$ the estimate is unbiased, cf. Zhang and Stephens (2009) and Zhang (2010). This estimate is modified in Zhang (2010) to reduce bias also for the case $k<-1$. For the most common shape parameters $k \geq-1$ both estimates are of the same value. We include the original ZSE in our simulation study.

However, for both variants, asymptotic normality is not established yet.

\section{Estimates of the fraction defective and their asymptotic normality}

Consider both tails separately, and assume now that the estimates $(\hat{\sigma}, \hat{k})$ in both tails, and therefore the estimates $\hat{p}_{L}$ and $\hat{p}_{U}$ are asymptotically normally distributed.

For the estimation of both the lower and upper parts of the fraction defective we use the smallest and largest $m$ observations, respectively. Since $q=q_{m} \rightarrow 0$ they are asymptotically independent and we get for the estimated fraction defective $\hat{p}=\hat{p}_{L}+\hat{p}_{U}$

$$
\sqrt{m} \frac{\hat{p}-p}{p} \rightarrow_{D} \mathcal{N}(0, W) \text { with } \quad W=\frac{p_{L}^{2} V\left(p_{L}\right)+p_{U}^{2} V\left(p_{U}\right)}{p^{2}},
$$

where $W$ depends on $p, p_{L}, p_{U}$ but also on $q$. For a discussion of the variance term, let us first consider the case of a one-sided specification limit. Recall that, for $k=0.5$ the asymptotic variances of the MLE and SWM are the same. For $k=-0.5$ the asymptotic variances of the MLE and LME are the same. Asymptotic variances for the MLE $\hat{p}$, and for densities with medium to long tails can be found in Kössler (1999), Table 3. We also have computed the asymptotic variances of the SWM for some short (lower) tail densities (Weibull, Beta, Gamma, Burr, all with various values of the parameter $k$ ), and found them similar to that for the MLE and for the Pareto with $k=-1$, except for the Gamma with $k=0.25$, and the Burr with $k=0.25$ or $k=0.5$.

An explanation of the exceptions may be that, at least for the MLE, the speed of convergence must be such that $\sqrt{m}\left(x_{U}-X_{(n-m)}\right) \rightarrow 0$ or $\sqrt{m}\left(X_{(m)}-x_{L}\right) \rightarrow 0$ which is faster than for other distributions, cf. Smith (1987), Theorem 5.1. However, 
if the ratios $\frac{p}{q}$ of the fraction defective $p$ and the used tail fraction $q$ are not too small then the dissimilarities are not so large.

Moreover, the asymptotic variances of the LME are also similar, for all densities considered, with the same exceptions as above.

Therefore, for the further investigation of the variance term we assume that both tails have the same type, especially the same GPD parameters $k$ and $\sigma$. Since, as we have seen, the asymptotic variances are rather similar for various distributions this assumption is no serious restriction. For each density we consider the one-sided $\left(p_{L}=0\right.$ or $\left.p_{U}=0\right)$ and the symmetric two-sided $\left(p_{L}=p_{U}\right)$ case and find that the asymptotic variances in the symmetric two-sided case are almost always slightly less than that in the one-sided cases.

Therefore, we may use a one-sided (larger) variance as we have done in designing our former sampling plans (cf. Kössler, 1999, 2015), and we are on the safe side. As in the former articles we choose the variance of the MLE from the Pareto and reverse Pareto, respectively, with $k=-1$. Note that, if the LME with the Pareto with $k=-\frac{1}{2}$ as the reference distribution were taken, then the resulting sampling plans (10)-(12) would be almost the same.

\section{Simulation Study}

As already mentioned, first simulations show that, for all the densities considered, the two estimates LME and ZSE are the best. These results are in accordance with that obtained for the GPD itself by other authors, cf. e.g. Zhang (2007), Zhang and Stephens (2009), and Mackay et al. (2011). Since we have established asymptotic normality for the LME with similar variances for the various underlying cdfs we might use it in the estimation of the fraction defective. However, for smaller sample sizes, it is biased and a correction of the acceptance number is necessary. For the LME we choose the corrected acceptance number $c_{\text {corr }}=c\left(1+\frac{3.3}{n}\right)$ which is found by simulation. Recall that the order of the correction term is the same as that obtained by Giles et al (2016) for the MLE of $(k, \sigma)$.

Although the asymptotic distribution of the ZSE is unknown our simulations indicate that the ZSE are unbiased. To check what happens if this estimate is used we include it in our simulation study.

In Table 1 the sampling plans $(n, m, c)$ given by (10)-(12) for twelve different 2 point conditions are presented. For comparison, the corresponding sample sizes $n_{V}$ and $n_{A}$ of the ordinary ML-variable sampling plan (which requires normality of the underlying population) and the attribute sampling plan, respectively, are given in the last two columns of Table 1 . The sample sizes for the ordinary ML variable sampling plan are computed by the R program ExLiebeRes of Krumbholz and Steuer (2014). The examples 1,2,4,5,7 and 8 are from Resnikoff (1952), example 10 is from Steland and Zähle (2009). To see whether the asymptotic theory works we have included an example with very large sample sizes (Example 12).

In an extensive simulation study, we investigate whether the two-point condition is maintained not only for the GPD but also for various distributions. 
Table 1 Sample sizes for the new and for the ordinary variable sampling plans together with that for the attribute sampling plan.

\begin{tabular}{c|cccc|rrr|rr}
\hline No. & $p_{1}$ & $1-\alpha$ & $p_{2}$ & $\beta$ & $n$ & $m$ & $c$ & $n_{V}$ & $n_{A}$ \\
\hline 1 & 0.0521 & 0.9500 & 0.1975 & 0.10 & 31 & 11 & 0.1088 & 27 & 45 \\
2 & 0.0634 & 0.9000 & 0.1975 & 0.10 & 31 & 11 & 0.1108 & 27 & 45 \\
3 & 0.0100 & 0.9000 & 0.0600 & 0.10 & 59 & 11 & 0.0241 & 36 & 88 \\
4 & 0.0100 & 0.9743 & 0.0592 & 0.10 & 80 & 13 & 0.0284 & 54 & 133 \\
5 & 0.0152 & 0.9000 & 0.0592 & 0.10 & 83 & 14 & 0.0296 & 54 & 111 \\
6 & 0.0100 & 0.9900 & 0.0600 & 0.10 & 90 & 14 & 0.0306 & 64 & 153 \\
7 & 0.0360 & 0.9500 & 0.0866 & 0.10 & 143 & 24 & 0.0580 & 106 & 189 \\
8 & 0.0406 & 0.9000 & 0.0866 & 0.10 & 149 & 25 & 0.0585 & 107 & 189 \\
9 & 0.0100 & 0.9900 & 0.0600 & 0.01 & 203 & 27 & 0.0238 & 111 & 263 \\
10 & 0.0200 & 0.9500 & 0.0500 & 0.05 & 316 & 34 & 0.0310 & 186 & 410 \\
11 & 0.0100 & 0.9900 & 0.0300 & 0.10 & 390 & 33 & 0.0199 & 217 & 590 \\
12 & 0.0200 & 0.9900 & 0.0300 & 0.01 & 4609 & 213 & 0.0244 & 2241 & 5362 \\
\hline
\end{tabular}

The simulation size is $M=2000$. The following cdfs are included in the simulation study: Pareto, Burr Type 12, all with various parameters, Cauchy (long tails), normal, logistic (medium tails), GPD with $k>0$, reverse Weibull, reverse Gamma, reverse Burr $\left(F(x)=1-\left(1-\frac{1}{x}\right)^{-\lambda}\right)$, and Beta, all with various parameters (short tails).

The asymptotic variances of the estimates of the fraction defective $p$ are almost the same for all densities. Therefore, for simplicity, in our simulation study we may assume that both tails have the same type. For example, the Pareto has long right tails, we added a part by reflecting the density on the $y$-axis, and dividing the result by 2 . This way both tails are long and of the same type. On the other hand, the reverse Weibull, for example, has a short right tail with the upper end point at zero. By reflecting the density somewhere where the cdf is almost vanishing, adding this part to the density, and dividing the result by some number such that we obtain a density, we arrive at a density with short left and right tail, both of the same Weibull type.

Of course, in practise, the tails may be of different types, then we expect intermediate behaviour, e.g. if the left tail is Weibull, and the right tail is Pareto then we may combine the simulation results for the Weibull and for the Pareto.

All computations are done by SAS programs.

To give an impression of the results we present OCs for the examples 8 and 12 which represent the cases of large and very large sample sizes. In Figures 1 and 2 we present the OC bands for two distributions, for the Pareto (with $k=-1$ ) and for the short tail Burr $(k=0.5)$. The figures for the other densities are omitted since they look very similar to that for the Pareto (with $k=-1$ ). The bands illustrate the various partitions of the fraction defective into a lower and upper part. The Pareto is chosen since it is the reference distribution, and the Burr represents a "bad" distribution, i.e. a distribution that behaves worst in our context. 

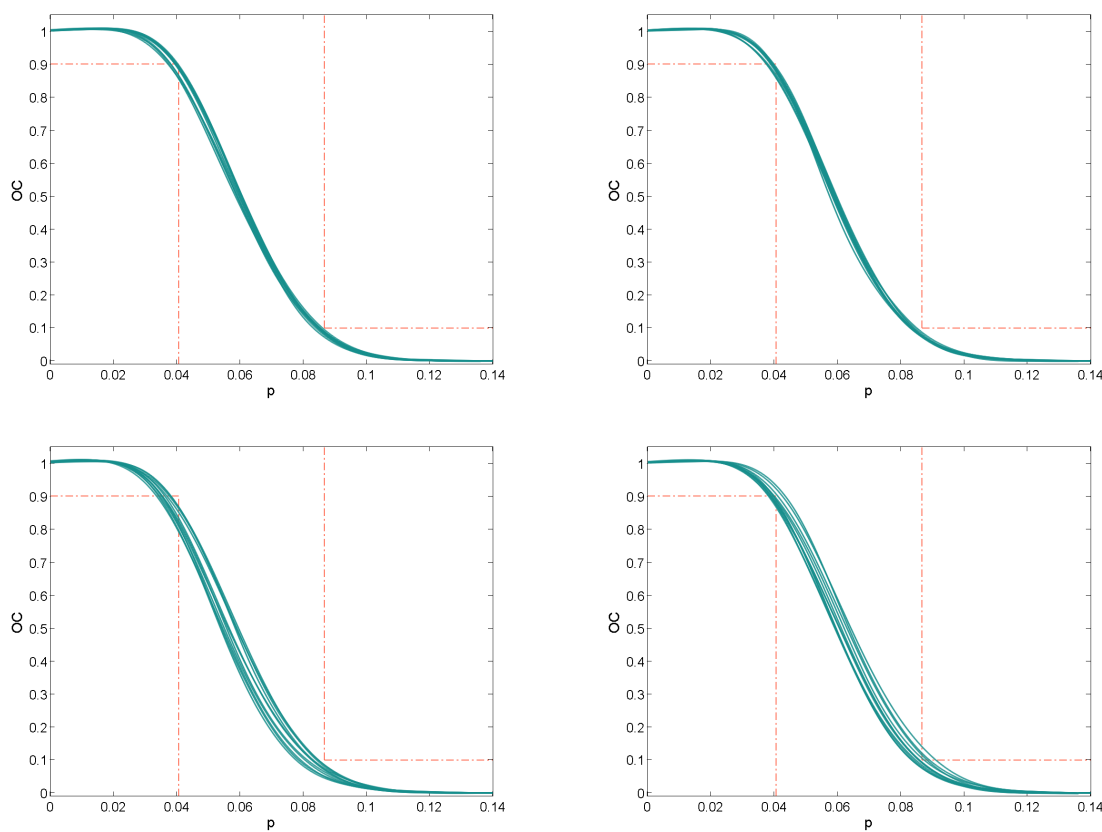

Fig. 1 Estimated operating characteristic for the new sampling plan, for example 8, left column: LME, right column: ZSE, first row Pareto $(k=-1)$, second row: Burr $(k=0.5)$ Recall that the sample size is $n=149$.

As we see in Figure 2, for very large sample sizes (Example 12) the two point condition is satisfied for all distributions considered. This way we have confirmed that our theory works, at least for very large sample sizes.

For the Pareto densities and for the Gaussian we have thin OC bands, for the Burr they are thicker where the two upper lines stay for the one-sided specification limits.

Consider Example 8, for the short-tail Burr the OC is bad, for the Pareto it is good, for the other distributions (not presented here) the results lie between these two extreme cases.

We also computed 0.95 -confidence regions for the parameters $\alpha$ and $\beta$ (based on the asymptotic normality) and found, for examples 12 and 8: for all medium and long tail densities the parameters are covered by the confidence region (altogether 44 cases each). For short-tail densities $(k=0.25, k=0.5)$ and for Example 12 in 21 of 26 cases $\alpha$ and $\beta$ are covered by the confidence interval if ZSE is used, for the LME it is only slightly worse. For example 8 the behaviour is, as expected, slightly worse. Interestingly, here LME is slightly better than ZSE. Perhaps the correction of the acceptance number works well. 

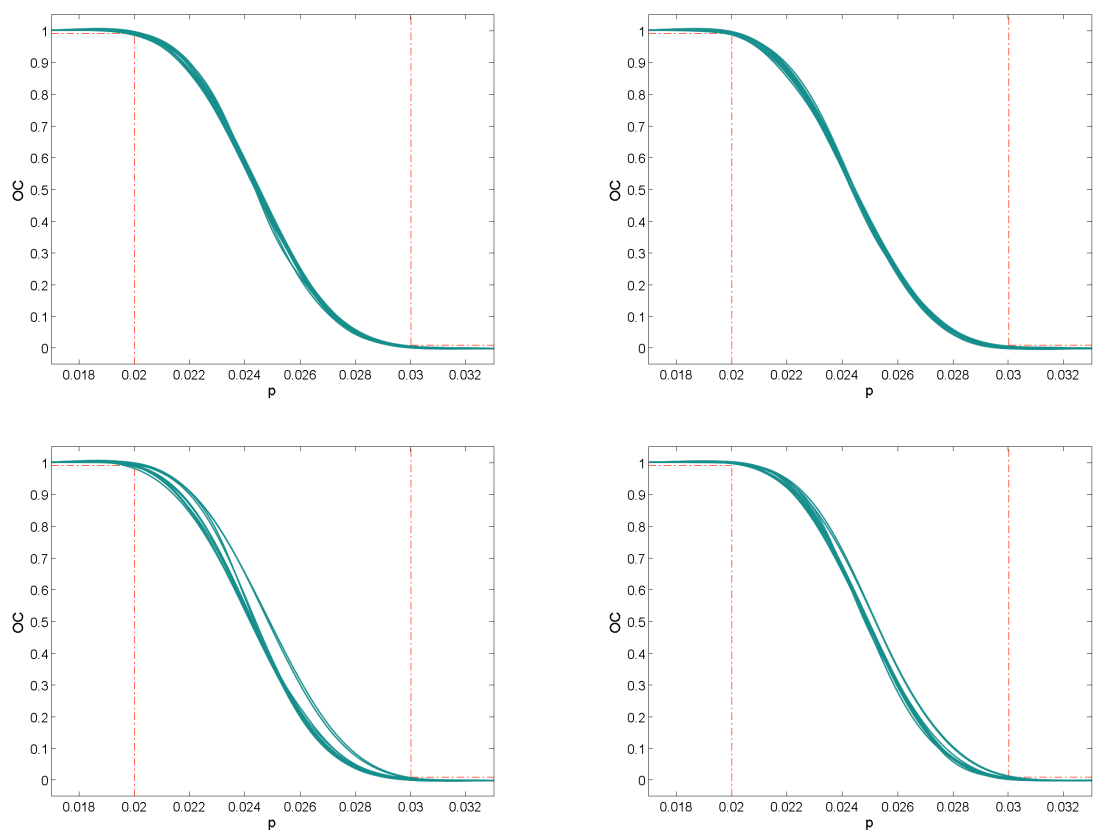

Fig. 2 Estimated operating characteristic for the new sampling plan, for example 12, left column: LME, right column: ZSE, first row Pareto $(k=-1)$, second row: Burr $(k=0.5)$ Recall that the sample size is $n=4609$.

\section{Illustrating example and discussion}

Let us illustrate our method by an example. Assume we have to inspect a lot of $1.5 \mathrm{~cm}$ glass fibres, and we assume that a unit should have a strength of at least $\mathrm{L}=0.75$. There is no upper specification limit, therefore we may set $U=\infty$. The two points $\left(p_{1}, 1-\alpha\right)$ and $\left(p_{2}, \beta\right)$, cf. the two-point condition (1) and (2), are given by $(0.01,0.90)$ and $(0.06,0.10)$, respectively. Then the resulting sampling plan is $(n, m, c)=(59,11,0.0241)$, cf. example 3 in Table 1 . Fortunately, we have a sample of size 63 at our hand that was provided by Smith and Naylor (1987), cf. also Hand et al (1994). These are four units more than necessary. This fact causes no problems since as $n$ increases $q$ decreases and the variance $W(p)$ becomes smaller, and the two-point conditions (1) and (2) are even more satisfied. We use the ZSE and obtain $\hat{p}=0.0324$ which is larger than the acceptance number $c=0.0241$, therefore the lot is to be rejected, at least if we insist on the fixed sampling plan.

Note that this data set was analysed by several authors, see e.g. Smith and Naylor (1987), Jones and Faddy (2003) and Fischer and Vaughan (2004), all using different distribution models. Rychlik and Rydén (2006) used the POT method, but with the PWM estimator for the estimation of quantiles. However, nobody investigated this data set in the quality control context.

If we are somewhat more flexible according a decision about accepting or rejecting the lot we may take different values of $m$ (or equivalently, of $q$ ), compute 

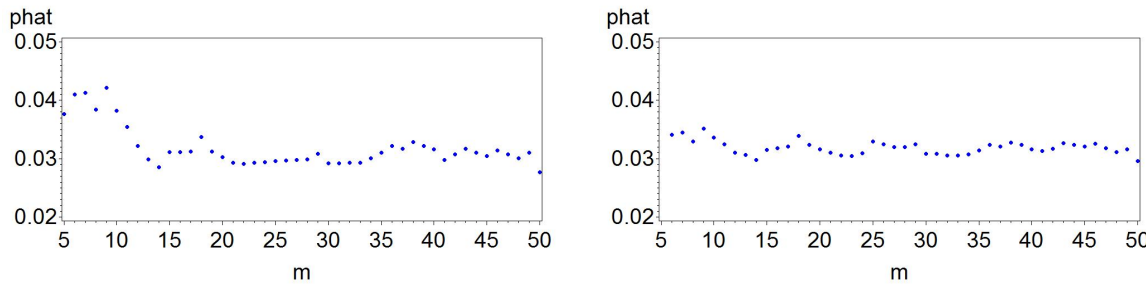

Fig. 3 Hill plot of $\hat{p}$ against $m$ for the glass fibres data set. The parameters of the GPD are estimated by the Likelihood moment (left figure) and Zhang-Stephens (right figure) method

the estimates $\hat{p}$, plot the pairs $(m, \hat{p})$, and look for "stable" estimates of $p$. This idea is similar to Hill plots where the estimate of the extreme value index $-\hat{k}$ is plotted against $m$, cf. e.g. Drees et al (2000).

For our example, we have computed both estimates, LME and ZSE, see the results in Figure 3, that for the LME and ZSE are on the left and right part, respectively. For $m \geq 12$ these estimates look relatively stable, we have values of $\hat{p}$ that lie around and slightly above the value 0.03 . Note that we used an empirically determined correction term for the LME because of bias $\left(\hat{p}:=\hat{p}\left(1+\frac{3.3}{n}\right)\right)$. In our example, we reject the lot for all choices of $m$, and we have "confirmed" our preliminary decision to reject the lot.

However, the case may not be so clear in instances where $\hat{p}(m)$ lies around the acceptance number $c$. Then the decision about accepting or rejecting the lot should be done after further discussions by an additional sample.

\section{Summary}

For normally distributed populations, of course, the LR- or ML-sampling plans are to be preferred. But usually, there is no exact information about the distribution of the underlying population in practice. Therefore, if the underlying cdf is continuous the proposed new variable sampling plan based on one of the LME or ZSM estimates should be applied instead of an attribute plan.

Since in the case of very light tails $(0.5 \leq k<1)$ the asymptotic normality of the LME is established only under a second order condition, we may suggest to take the SWM estimator in the case of $k>0.5$ (cf. Kössler, 2015).

Acknowledgements The authors like to thank the referees for their valuable comments that lead to an improvement of the presentation.

\section{References}

Balkema A, de Haan L (1974) Residual life time at great age. Annals of Probability 2:792-804 
Beirlant J, Goegebeur Y, Segers J, Teugels J (2004) Statistics of Extremes, Theory and Applications. Wiley, Chichester

Beirlant J, Caero F, Gomes Y (2012) An overview and open research topics in statistics of univariate extremes. REVSTAT- Statistical Journal pp 1-31

Bruhn-Suhr M, Krumbholz W (1990) A new variable sampling plan for normally distributed lots with unknown standard deviation and double specification limits. Statistische Hefte 31:195-207

Bruhn-Suhr M, Krumbholz W (1991) Exact two-sided Lieberman-Resnikoff sampling plans. Statistische Hefte 32:233-241

Caeiro F, Gomes MI (2015) Threshold selection in extreme value analysis. In: Extreme Value Modeling and Risk Analysis: Methods and Applications, eds. Dipak Dey and Yun Yan, Chapman and Hall/CRC, pp 69-87

Castillo E, Hadi AS (1997) Fitting the Generalized Pareto distribution to data. Journal of the American Statistical Association 92:1609-1620

Davison AC (1984) Modeling excesses over high threshold with an application. In: Statistical Extremes and Applications, ed. J. Tiago de Oliveira, D. Reidel, pp 461482

Drees H, De Haan L, Resnick S (2000) How to make a Hill plot. The Annals of Statistics 28:254-274

Falk M (1989) Best attainable rate of joint convergence of extremes. In: Extreme value theory, ed. J. Hüsler, R.-D.Reiss, Proceedings of a conference held in Oberwolfach, Dec.6-12, 1987, Springer, pp 1-9

Fischer M, Vaughan D (2004) The beta-hyperbolic secant (bhs) distribution. Diskussionspapiere, Friedrich-Alexander-Universität Erlangen-Nürnberg, Lehrstuhl für Statistik und Ökonometrie

Giles D, Feng H, Godwin R (2016) Bias-corrected Maximum Likelihood estimation of the parameters of the Generalized pareto distribution. Communications in Statistics Theory and Methods 45:2465 - 2483

Grimshaw SD (1993) Computing Maximum Likelihood estimates for the generalized Pareto distribution. Technometrics 35:185-191

de Haan L, Fereira A (2006) Extreme Value Theory. Springer, New York

Hand D, Daly F, Dunn A, McConway K, Ostrowski E (1994) Handbook of Small Data Sets. Chapman and Hall, London

Hosking J, Wallis J (1987) Parameter and quantile estimation for the generalized Pareto distribution. Technometrics 39:339-349

Jones M, Faddy M (2003) A skew extension of the t-distribution, with applications. Journal of the Royal Statistical Society, Series B 65(1):159-174

Kössler W (1999) A new one-sided variable inspection plan for continuous distribution functions. Allgemeines Statistisches Archiv 83:416-433

Kössler W (2015) Variable inspection plans for continuous distributions with unknown short tail distributions. In: Stochastik Models, Statistics, and Their Applications, eds. A. Steland, E. Rafajlowicz, K. Szajowski, Springer, Heidelberg, pp 93-100

Kössler W, Lenz HJ (1995) On the robustness of Lieberman-Resnikoff sampling plans by variables. Journal of the Indian Association for Productivity, Quality and Reliability 20:93-105 
Kössler W, Lenz HJ (1997) On the non-robustness of Maximum-Likelihood sampling plans by variables. In: Frontiers in Statistical Quality Control 5, ed.H.-J.Lenz, P.Th.Wilrich, Physica, Heidelberg, pp 38-52

Kössler W, Lenz B, Lenz HJ (1994) Exvar - exact variable inspection plans in statistical quality control. Statistical Software Newsletter 17:97-99

Krumbholz W, Steuer D (2014) On exact and optimal single sampling plans by variables. Advances in Statistical Analysis 98:87-101

Lieberman G, Resnikoff G (1955) Sampling plans for inspection by variables. Journal of the American Statistical Association 50:457-516

Mackay EB, Challenor PG, Bahaj AS (2011) A comparison of estimators for the generalized Pareto distribution. Ocean Engeneering 38:1338-1346

Ott J (2016) Annahmeprüfung für beliebige stetige Verteilungen. Diploma thesis, Humboldt Universität zu Berlin

Pickands J (1975) Statistical inference using extreme order statistics. Annals of Statistics 3:119-135

Resnikoff G (1952) A new two-sided acceptance region for sampling by variables. Technical Report 8, Applied mathematics and Statistics Laboratory, Stanford University

Rychlik I, Rydén J (2006) Probability and Risk Analysis: An Introduction for Engineers. Springer, Heidelberg

Smith R (1987) Estimating tails of probability distributions. Annals of Statistics 15:1174-1207

Smith R, Naylor J (1987) A comparison of Maximum Likelihood and Bayesian estimators for the three-parameter Weibull distribution. Applied Statistics 36(3):358 369

Smith R, Weissman I (1985) Maximum Likelihood estimation of the lower tail of a probability distribution. Journal of the Royal Statistical Society B 47:285-298

Steland A, Zähle H (2009) Sampling inspection by variables: nonparametric setting. Statistica Neerlandica 63:101-123

Uhlmann W (1982) Statische Qualitätskontrolle. Teubner, Stuttgart

Zhang J (2007) Likelihood moment estimation for the Generalized Pareto distribution. Australian and New Zealand Journal of Statistics 49:69-77

Zhang J (2010) Improving an estimation for the Generalized Pareto distribution. Technometrics 52:335-339

Zhang J, Stephens MA (2009) A new and efficient estimation method for the Generalized Pareto Distribution. Technometrics 51:316-325

Zhou C (2009) Existence and consistency of the maximum likelihood estimator for the extreme value index. Journal of Multivariate Analysis 100:794-815

Zhou C (2010) The extent of the maximum likelihood estimator for the extreme value index. Journal of Multivariate Analysis 101:971-983 